\title{
Aspects of Control for a Parafoil and Payload System
}

\author{
Nathan Slegers* and Mark Costello ${ }^{\dagger}$ \\ Oregon State University, Corvallis, Oregon 97331
}

\begin{abstract}
A parafoil controlled by parafoil brake deflection offers a lightweight and space-efficient control mechanism for autonomous placement of air-dropped payloads to specified ground coordinates. The work reported here investigates control issues for a parafoil and payload system with left and right parafoil brakes used as the control mechanism. It is shown that parafoil and payload systems can exhibit two basic modes of lateral control, namely, roll and skid steering. These two modes of lateral steering generate lateral response in opposite directions. For example, a roll steer configuration turns left when the right parafoil brake is activated, whereas a skid steer configuration turns right under the same control input. In transition between roll and skid lateral steering, the lateral response is zero, and the system becomes uncontrollable. Angle of incidence, canopy curvature of the parafoil, and magnitude of brake deflections are important design parameters for a controllable parafoil and payload system and greatly effect control response, including whether the basic lateral control mode is roll or skid steering. It is shown how the steering mode switches when fundamental design parameters are altered and as the magnitude of the brake deflection increases. The mode of directional control transitions toward roll steering as the canopy curvature decreases or the angle of incidence becomes more negative. The mode of directional control transitions away from the roll steering mode as the magnitude of the brake deflection increases, and for "large" brake deflections most parafoils will always skid steer.
\end{abstract}

\section{Nomenclature}

$A_{b}$

$A_{i}$

$C_{D}^{b}$

$C_{\mathrm{Di}}^{p}$

$C_{\mathrm{Li}}$

$C_{l_{0}}, C_{m_{0}}, C_{n_{0}}$

$C_{l_{\delta}}, C_{m_{\delta}}, C_{n_{\delta}}$

$C_{x_{0}}, C_{y_{0}}, C_{z_{0}}$
$C_{x_{\delta}}, C_{y_{\delta}}, C_{z \delta}$

$C_{x_{\delta}}, C_{y_{\delta}}, C_{z \delta}$
$F_{A}^{b}, F_{A}^{p}$

$F_{\mathrm{xc}}, F_{\mathrm{yc}}, F_{\mathrm{zc}}$

$I_{b}, I_{p}$

$I_{F}, I_{M}$

$K_{c}, C_{c}$

$M_{A}$

$M_{\mathrm{UA}}$

$M_{\mathrm{xc}}, M_{\mathrm{yc}}, M_{\mathrm{zc}}$

$m_{b}, m_{p}$

$p_{b}, q_{b}, r_{b}$

$p_{p}, q_{p}, r_{p}$

$=$ payload reference area

$=$ drag coefficient of payload in their respective frames in an inertial frame coefficient matrices coefficients of joint $C$ aerodynamic forces aerodynamic forces in an inertial frame

$=$ reference area of $i$ th panel of parafoil canopy

$=$ drag coefficient of $i$ th panel of parafoil canopy

$=$ lift coefficient of $i$ th panel of parafoil canopy

$=$ rolling, pitching, and yawing moment coefficients from no brake deflection

$=$ rolling, pitching, and yawing moment coefficients from a brake deflection

$=$ force coefficients from no brake deflection

$=$ force coefficients from a brake deflection

$=$ aerodynamic force on payload and parafoil

$=$ components of joint constraint force

$=$ inertia matrix of payload and parafoil

$=$ apparent mass force and moment

$=$ rotational stiffness and damping

$=$ moment on parafoil caused by steady

$=$ moment on payload caused by unsteady

$=$ components of joint constraint moment

$=$ mass of payload and parafoil

$=$ components of angular velocity of payload in payload reference frame $(b)$

$=$ components of angular velocity of parafoil in parafoil reference frame $(p)$

\footnotetext{
Received 15 August 2002; revision received 8 November 2002; accepted
or publication 7 April 2003. Copyright (C) 2003 by the American Institute of

Received 15 August 2002; revision received 8 November 2002; accepted
for publication 7 April 2003. Copyright (C) 2003 by the American Institute of Aeronautics and Astronautics, Inc. All rights reserved. Copies of this paper may be made for personal or internal use, on condition that the copier pay the $\$ 10.00$ per-copy fee to the Copyright Clearance Center, Inc., 222 Rosewood Drive, Danvers, MA 01923; include the code 0731-5090/03 \$10.00 in correspondence with the CCC.

* Graduate Research Assistant, Department of Mechanical Engineering. Member AIAA.

${ }^{\dagger}$ Assistant Professor, Department of Mechanical Engineering. Member AIAA.
}

$T_{b}$

$T_{i}$

$T_{p}$

$T_{t_{i}}$

$u_{A}, v_{A}, w_{A}$

$u_{b}, v_{b}, w_{b}$

$u_{i}, v_{i}, w_{i}$

$V_{s}$

$x, y, z$

$\dot{x}, \dot{y}, \dot{z}$

$x_{\text {ca }}, y_{\text {ca }}, z_{\text {ca }}$

$x_{\mathrm{cb}}, y_{\mathrm{cb}}, z_{\mathrm{cb}}$

$x_{\mathrm{cp}}, y_{\mathrm{cp}}, z_{\mathrm{cp}}$

$x_{\mathrm{pa}}, y_{\mathrm{pa}}, z_{\mathrm{pa}}$

$\eta$

$\phi_{b}, \theta_{\sim}, \psi_{\sim}$

$\dot{\phi}_{b}, \tilde{\theta}_{b}, \psi_{b}$

$\phi_{\tilde{\phi}}, \theta_{p}, \psi_{p}$

$\tilde{\phi}_{p}, \tilde{\theta}_{p}, \tilde{\psi}_{p}$

$\psi_{t_{i}}$
$=$ transformation matrix from inertial reference frame to payload reference frame

$=$ transformation matrix from $i$ th panel's reference frame to parafoil reference frame

$=$ transformation matrix from inertial reference frame to parafoil reference frame

$=$ transformation matrix from inertial reference frame to $i$ th command trajectory reference frame

$=$ components of relative air velocity of apparent mass center in parafoil reference frame

$=$ components of relative air velocity of mass center of payload in payload reference frame

$=$ components of relative air velocity of aerodynamic center of panel $i$ in $i$ th frame

$=$ magnitude of velocity vector of mass center of payload

$=$ components of position vector of point $\mathrm{C}$ in an inertial frame

$=$ components of velocity vector of point $\mathrm{C}$ in an inertial frame

$=$ components of vector from point $\mathrm{C}$ to apparent mass center in parafoil reference frame

$=$ components of vector from point $\mathrm{C}$ to mass center of payload in payload reference frame

$=$ components of vector from point $\mathrm{C}$ to mass center of parafoil in parafoil reference frame

$=$ components of vector from parafoil mass center to apparent mass center in parafoil reference frame

$=$ angle of incidence

$=$ Euler roll, pitch, and yaw angles of payload

$=$ payload Euler roll, pitch, and yaw angles for roll constraint moment computation

$=$ Euler roll, pitch, and yaw angles of parafoil

$=$ parafoil Euler roll, pitch, and yaw angles for roll constraint moment computation

$=$ angle between inertial reference frame and $i$ th command trajectory reference frame

\section{Introduction}

O produce rapidly deployableand ready fighting units, weapon system developers have recognized the driving need to quickly 
station large numbers of soldiers, along with their equipment in low density over a large land area. Use of this troop and equipment deployment strategy requires autonomous air delivery of many individual equipment packages to specific rendezvous points. One concept to realize this goal is to equip each individual package with a parafoil and inexpensive guidance and control module so that each package can steer itself to a prespecified rendezvous point after release from a delivery aircraft.

Detailed dynamic simulation of the flight mechanics of parachute and load systems appears to have commenced with the work of Wolf, who considered the stability of a parachute connected to a load. ${ }^{1}$ Using a 10-degree-of-freedom(DOF) representation, Wolf established that stability is reduced as riser length is increased or parachute weight is increased and that stability is improved by increasing parachute axial and normal aerodynamic force. Later, Doherr and Schilling reported on the development of a nine-DOF dynamic model. ${ }^{2}$ By comparing results from six- and nine-DOF models, they conclude a nine-DOF model adequately predicts stability characteristics. Furthermore, their work established the sensitivity of the motion of a parachute and load system to atmospheric winds. Hailiang and Zizeng used a nine-DOF model to study the motion of a parafoil and payload system. ${ }^{3}$ In contrast to Doherr and Schilling, they reported only small differences in the motion and stability between six- and nine-DOF dynamic models. ${ }^{2}$ In studying

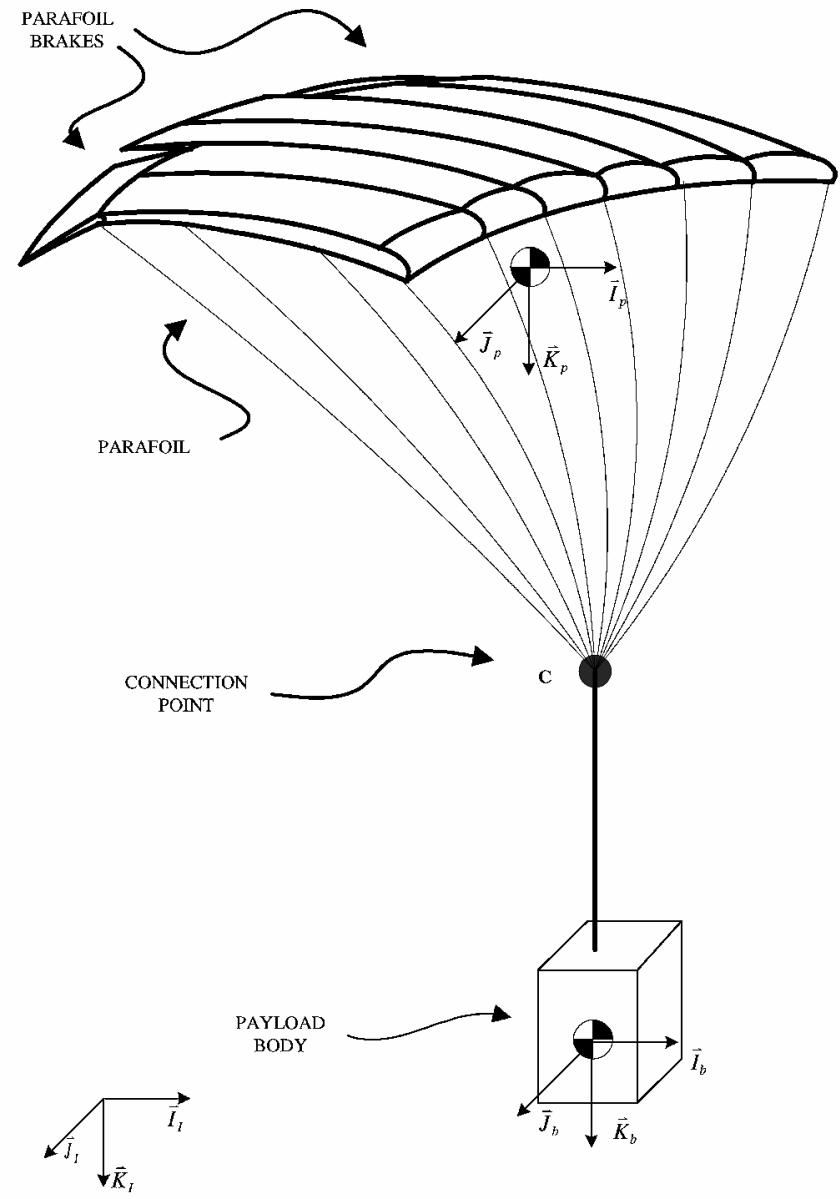

Fig. 1 Parafoil and payload system. stability characteristics as a function of the pitch inertia of the payload, Hailiang and Zizeng found the decay ratio and period increase as pitch inertiais increased. ${ }^{3}$ Iosilevskii established center of gravity and lift coefficient limits for a gliding parachute. ${ }^{4}$ Brown analyzed the effects of scale and wing loading on a parafoil using a linearized model based on computer calculated aerodynamic coefficients. ${ }^{5}$ Brown found that steady-state turn response of small parafoils is more sensitive to control inputs than larger parafoils. More recent efforts by Zhu et al. as well as Gupta et al. have incorporated parafoil structural dynamics into the dynamic model of a parachute and payload system. ${ }^{6,7}$ A significant amount of literature has been amassed in the area of experimental parafoil dynamics beginning with Ware and Hassell who investigatedram-air parachutes in a wind tunnel by varying wing area and wing chord. ${ }^{8}$ More recently, extensive flight tests have been reported on NASA's X-38 parafoil providing steadystate data and aerodynamics for large-scale parafoils. ${ }^{9,10}$ This paper considers a payload that has an attached parafoil with brakes used as the control mechanism. Using a dynamic modeling approach similar to Doherr and Schilling and Hailiang and Zizeng, stability and control characteristics of this system are examined.$^{2,3}$ Particular attention is paid to steady-state control response as a function of fundamental design parameters such as parafoil canopy geometry, angle of incidence, and varying control deflection.

\section{Parafoil and Payload Dynamic Model}

Figure 1 shows a schematic of the dynamic system that consists of a payload body connected to a parafoil canopy. A constant velocity joint couples the parafoil and payload components at point $\mathrm{C}$. The inertial frame shown in Fig. 1 is fixed to the surface of the Earth. With the exception of movable parafoil brakes, the parafoil canopy is considered to be a fixed shape once it has completely inflated. Figures 2 and 3 show a schematic of the parafoil canopy geometry. Connected to each panel are brakes that change the aerodynamic loads on the parafoil when they are deflected. The parafoil canopy is connected to joint $\mathrm{C}$ by a rigid massless link from the mass center of the canopy. The payload is connected to joint $\mathrm{C}$ by a rigid massless link from the mass center of the payload. Both the parafoil and the payload are free to rotate about joint $\mathrm{C}$ but are constrained by the force and moment at the joint. The combined system of the

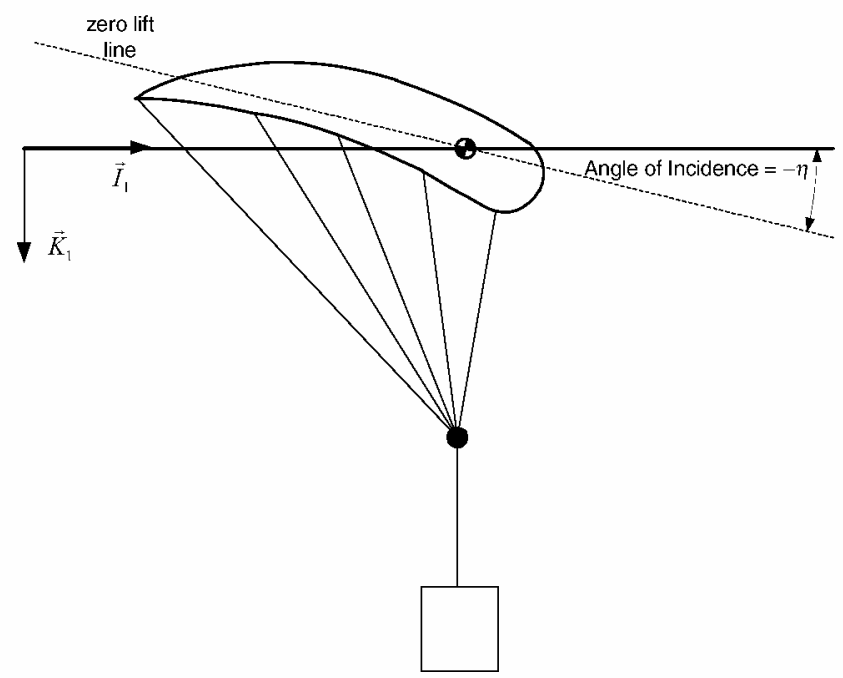

Fig. 3 Angle of incidence.

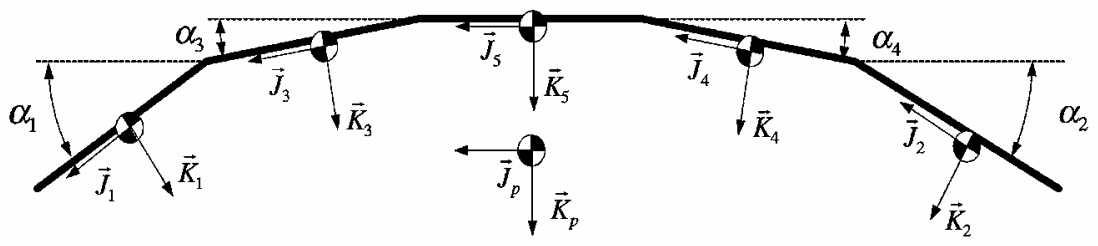

Fig. 2 Parafoil canopy geometry. 
parafoil canopy and the payload are modeled with nine DOF, including three inertial position components of the joint $\mathrm{C}$ as well as the three Euler orientation angles of the parafoil canopy and the payload. The kinematic equations for the parafoil canopy and the payload are provided in Eqs. (1-3):

$$
\begin{gathered}
\left\{\begin{array}{l}
\dot{x}_{c} \\
\dot{y}_{c} \\
\dot{z}_{c}
\end{array}\right\}=\left\{\begin{array}{c}
u_{c} \\
v_{c} \\
w_{c}
\end{array}\right\} \\
\left\{\begin{array}{c}
\dot{\phi}_{b} \\
\dot{\theta}_{b} \\
\dot{\psi}_{b}
\end{array}\right\}=\left[\begin{array}{ccc}
1 & s_{\phi_{b}} t_{\theta_{b}} & c_{\phi_{b}} t_{\theta_{b}} \\
0 & c_{\phi_{b}} & -s_{\phi_{b}} \\
0 & s_{\phi_{b}} / c_{\theta_{b}} & c_{\phi_{b}} / c_{\theta_{b}}
\end{array}\right]\left\{\begin{array}{l}
p_{b} \\
q_{b} \\
r_{b}
\end{array}\right\} \\
\left\{\begin{array}{c}
\dot{\phi}_{p} \\
\dot{\theta}_{p} \\
\dot{\psi}_{p}
\end{array}\right\}=\left[\begin{array}{ccc}
1 & s_{\phi_{p}} t_{\theta_{p}} & c_{\phi_{p}} t_{\theta_{p}} \\
0 & c_{\phi_{p}} & -s_{\phi_{p}} \\
0 & s_{\phi_{p}} / c_{\theta_{p}} & c_{\phi_{p}} / c_{\theta_{p}}
\end{array}\right]\left\{\begin{array}{l}
p_{p} \\
q_{p} \\
r_{p}
\end{array}\right\}
\end{gathered}
$$

The dynamic equations are formed by first separating the system at the coupling joint, then exposing the joint constraint force and moment acting on both bodies. The translational and rotational dynamics are inertially coupled because the position degrees of freedom of the system are the inertial position vector components of the coupling joint. The constraint force is a quantity of interest to monitor during the simulation so that it is retained in the dynamic equations rather than being algebraically eliminated. Equation (4) represents the translationaland rotationaldynamic equations of both the parafoil and payload concatenated into matrix form:

$\left[\begin{array}{cccc}-m_{b} S_{c}^{b} & 0 & m_{b} T_{b} & -T_{b} \\ 0 & -I_{F} S_{c}^{a}-m_{p} S_{c}^{p} & I_{F} T_{p}+m_{p} T_{p} & T_{p} \\ I_{b} & 0 & 0 & S_{c}^{b} T_{b} \\ 0 & I_{M}+I_{p}-S_{p}^{a} I_{F} S_{c}^{a} & S_{p}^{a} I_{F} T_{p} & -S_{c}^{p} T_{p}\end{array}\right]$

$$
\times\left\{\begin{array}{c}
\dot{p}_{b} \\
\dot{q}_{b} \\
\dot{r}_{b} \\
\cdots \\
\dot{p}_{p} \\
\dot{q}_{p} \\
\dot{r}_{p} \\
\cdots \\
\ddot{x}_{c} \\
\ddot{y}_{c} \\
\ddot{z}_{c} \\
\cdots \\
F_{\mathrm{xc}} \\
F_{\mathrm{yc}} \\
F_{\mathrm{zc}}
\end{array}\right\}=\left\{\begin{array}{l}
B_{1} \\
B_{2} \\
B_{3} \\
B_{4}
\end{array}\right\}
$$

The matrix in Eq. (4) is a block $4 \times 4$ matrix, where each element is a $3 \times 3$ matrix. Rows 1-3 in Eq. (4) are forces acting on the payload mass center expressed in the payload frame, and rows 7-9 are the moments about the payload mass center also in the payload frame. Rows 4-6 in Eq. (4) are forces acting on the parafoil mass center expressed in the parafoil frame, and rows 10-12 are the moments about the parafoil mass center also in the parafoil frame. The $S_{i}^{j}$ matrices are cross-product operator matrices, working on different vectors from $i$ to $j$ associated with the system configuration.

$$
S_{i}^{j}=\left[\begin{array}{ccc}
0 & -z_{i j} & y_{i j} \\
z_{i j} & 0 & -x_{i j} \\
-y_{i j} & x_{i j} & 0
\end{array}\right]
$$

The matrix $T_{b}$ represents the transformation matrix from an inertial reference frame to the payload reference frame,

$$
T_{b}=\left[\begin{array}{ccc}
c_{\theta_{b}} c_{\psi_{b}} & c_{\theta_{b}} s_{\psi_{b}} & -s_{\theta_{b}} \\
s_{\phi_{b}} s_{\theta_{b}} c_{\psi_{b}}-c_{\phi_{b}} s_{\psi_{b}} & s_{\phi_{b}} s_{\theta_{b}} s_{\psi_{b}}+c_{\phi_{b}} c_{\psi_{b}} & c_{\theta_{b}} s_{\phi_{b}} \\
c_{\phi_{b}} s_{\theta_{b}} c_{\psi_{b}}+s_{\phi_{b}} s_{\psi_{b}} & c_{\phi_{b}} s_{\theta_{b}} s_{\psi_{b}}-s_{\phi_{b}} c_{\psi_{b}} & c_{\phi_{b}} c_{\theta_{b}}
\end{array}\right]
$$

whereas, $T_{p}$ represents the transformation matrix from an inertial reference frame to the parafoil reference frame.

$$
T_{p}=\left[\begin{array}{ccc}
c_{\theta_{p}} c_{\psi_{p}} & c_{\theta_{p}} s_{\psi_{p}} & -s_{\theta_{p}} \\
s_{\phi_{p}} s_{\theta_{p}} c_{\psi_{p}}-c_{\phi_{p}} s_{\psi_{p}} & s_{\phi_{p}} s_{\theta_{p}} s_{\psi_{p}}+c_{\phi_{p}} c_{\psi_{p}} & c_{\theta_{p}} s_{\phi_{p}} \\
c_{\phi_{p}} s_{\theta_{p}} c_{\psi_{p}}+s_{\phi_{p}} s_{\psi_{p}} & c_{\phi_{p}} s_{\theta_{p}} s_{\psi_{p}}-s_{\phi_{p}} c_{\psi_{p}} & c_{\phi_{p}} c_{\theta_{p}}
\end{array}\right]
$$

The common shorthand notation for trigonometric functions is employed, where $\sin (\alpha) \equiv s_{\alpha}, \cos (\alpha) \equiv s_{\alpha}$, and $\tan (\alpha) \equiv t_{\alpha}$. The matrices $I_{b}$ and $I_{p}$ represent the mass moment of inertia matrices of the payload and the parafoil body with respect to their respective mass centers, and the matrices $I_{F}$ and $I_{M}$ represent the apparent mass force coefficient matrix and apparent mass moment coefficient matrix respectively:

$$
\begin{aligned}
I_{F} & =\left[\begin{array}{lll}
A & 0 & 0 \\
0 & B & 0 \\
0 & 0 & C
\end{array}\right] \\
I_{M} & =\left[\begin{array}{ccc}
I_{A} & 0 & 0 \\
0 & I_{B} & 0 \\
0 & 0 & I_{C}
\end{array}\right]
\end{aligned}
$$

Equations (11-14) provide the right-hand side vector of Eq. (4).

$$
\begin{aligned}
& B_{1}=W_{b}+F_{A}^{b}-m_{b} S_{w}^{b} S_{w}^{b}\left\{\begin{array}{l}
x_{\mathrm{cb}} \\
y_{\mathrm{cb}} \\
z_{\mathrm{cb}}
\end{array}\right\} \\
& B_{2}=W_{p}+F_{A}^{p}-I_{F} \dot{T}_{p}\left\{\begin{array}{l}
\dot{x} \\
\dot{y} \\
\dot{z}
\end{array}\right\}-S_{\omega}^{p} I_{F}\left\{\begin{array}{c}
u_{A} \\
v_{A} \\
w_{A}
\end{array}\right\}-m_{p} S_{w}^{p} S_{w}^{p}\left\{\begin{array}{l}
x_{\mathrm{cp}} \\
y_{\mathrm{cp}} \\
z_{\mathrm{cp}}
\end{array}\right\} \\
& B_{3}=M_{c}-S_{w}^{b} I_{b}\left\{\begin{array}{l}
p_{b} \\
q_{b} \\
r_{b}
\end{array}\right\} \\
& B_{4}=M_{A}-T_{p} T_{b}^{T} M_{c}-S_{w}^{p}\left(I_{p}+I_{M}\right)\left\{\begin{array}{c}
p_{p} \\
q_{p} \\
r_{p}
\end{array}\right\} \\
& -S_{p}^{a} I_{F} \dot{T}_{p}\left\{\begin{array}{l}
\dot{x} \\
\dot{y} \\
\dot{z}
\end{array}\right\}-S_{p}^{a} S_{\omega}^{p} I_{F}\left\{\begin{array}{l}
u_{A} \\
v_{A} \\
w_{A}
\end{array}\right\}
\end{aligned}
$$

where

$$
\begin{aligned}
S_{w}^{b} & =\left[\begin{array}{ccc}
0 & -r_{b} & q_{b} \\
r_{b} & 0 & -p_{b} \\
-q_{b} & p_{b} & 0
\end{array}\right] \\
S_{w}^{p} & =\left[\begin{array}{ccc}
0 & -r_{p} & q_{p} \\
r_{p} & 0 & -p_{p} \\
-q_{p} & p_{p} & 0
\end{array}\right]
\end{aligned}
$$

The weight force vectors on both the parafoil and payload in their respective body axes are given in Eqs. (16) and (17). 


$$
\begin{gathered}
W_{b}=m_{b} g\left\{\begin{array}{c}
-s_{\theta_{b}} \\
s_{\phi_{b}} c_{\theta_{b}} \\
c_{\phi_{b}} c_{\theta_{b}}
\end{array}\right\} \\
W_{p}=m_{P} g\left\{\begin{array}{c}
-s_{\theta_{p}} \\
s_{\phi_{p}} c_{\theta_{p}} \\
c_{\phi_{p}} c_{\theta_{p}}
\end{array}\right\}
\end{gathered}
$$

Equation (18) gives aerodynamic force on the payload from drag, which acts at the center of pressure of the payload assumed to be located at the payload's center.

$$
F_{A}^{b}=-\frac{1}{2} \rho A_{b} V_{b} C_{D}^{b}\left\{\begin{array}{c}
u_{b} \\
v_{b} \\
w_{b}
\end{array}\right\}
$$

The payload frame components of the payload's mass center velocity that appear in Eq. (18) are computed using Eq. (19).

$$
\left\{\begin{array}{c}
u_{b} \\
v_{b} \\
w_{b}
\end{array}\right\}=T_{b}\left\{\begin{array}{c}
\dot{x} \\
\dot{y} \\
\dot{z}
\end{array}\right\}+S_{w}^{b}\left\{\begin{array}{c}
\rho_{x}^{b} \\
\rho_{y}^{b} \\
\rho_{z}^{b}
\end{array}\right\}
$$

The shape of the parafoil canopy is modeled by joining panels of the same cross section side by side at angles with respect to a horizontal plane. The $i$ th panel of the parafoil canopy experiences lift and drag forces that are modeled using Eqs. (20) and (21), where $u_{i}, v_{i}, w_{i}$ are the velocity components of the center of pressure of the $i$ th canopy panel in the $i$ th canopy panel frame. ${ }^{11}$

$$
\begin{gathered}
L_{i}=\frac{1}{2} \rho A_{i} \sqrt{u_{i}^{2}+w_{i}^{2}} C_{\mathrm{Li}}\left\{\begin{array}{c}
w_{i} \\
0 \\
-u_{i}
\end{array}\right\} \\
D_{i}=-\frac{1}{2} \rho A_{i} V_{i} C_{\mathrm{Di}}^{p}\left\{\begin{array}{c}
u_{i} \\
v_{i} \\
w_{i}
\end{array}\right\}
\end{gathered}
$$

Equation (20) provides the total aerodynamic force on the parafoil canopy.

$$
F_{A}=\sum_{i=1}^{n} T_{i}\left(L_{i}+D_{i}\right)
$$

The opening of the parafoil is modeled as the area increasing nonlinearly over time similar to the approach taken by Wolfe and Peterson. ${ }^{12}$ When the parafoil is released from its pack, each panel area $A_{i}$ of the parafoil is small and increasesover time until reaching the final panel area. The increase in panel area is modeled as a known nonlinear function. Computationally the panel area is obtained at an arbitrary time by linear interpolation of a table of data. This approach is not meant to completely model the complicated process of parafoil inflation but rather provide a realistic initial disturbance.

The applied moment about the parafoil's mass center contains contributions from the steady aerodynamic forces and the coupling joint's resistance to twisting. The moment caused by a panel's steady aerodynamic forces is computed with a cross product between the distance vector from the mass center of the parafoil to the center of pressure of the panel and the force itself. Equation (23) gives the total moment from the steady aerodynamic forces:

$$
M_{A}=\sum_{i=1}^{n} S_{p}^{C P_{i}} T_{i}\left(L_{i}+D_{i}\right)
$$

where

$$
T_{i}=\left[\begin{array}{ccc}
1 & 0 & 0 \\
0 & c_{\alpha_{i}} & -s_{\alpha_{i}} \\
0 & s_{\alpha_{i}} & c_{\alpha_{i}}
\end{array}\right]
$$

The resistance to twisting of the coupling joint is modeled as a rotational spring and damper given by Eq. (25):

$$
M_{c}=\left\{\begin{array}{c}
0 \\
0 \\
K_{c}\left(\tilde{\psi}_{p}-\tilde{\psi}_{b}\right)+C_{c}\left(\dot{\tilde{\psi}}_{p}-\dot{\tilde{\psi}}_{b}\right)
\end{array}\right\}
$$

The angles $\tilde{\psi}_{p}$ and $\tilde{\psi}_{b}$ are the modified Euler yaw angles of the parafoil and payload that come from a modified sequence of rotations where the Euler yaw angle is the final rotation. The Euler yaw angles $\tilde{\psi}_{p}$ and $\tilde{\psi}_{b}$ for the modified sequence of rotations can be related to the original Euler angles by Eqs. (26) and (27):

$$
\begin{aligned}
& \tilde{\psi}_{p}=\tan ^{-1}\left(\frac{s_{\phi_{p}} s_{\theta_{p}} c_{\psi_{p}}-c_{\phi_{p}} s_{\psi_{p}}}{c_{\theta_{p}} c_{\psi_{p}}}\right) \\
& \tilde{\psi}_{b}=\tan ^{-1}\left(\frac{s_{\phi_{b}} s_{\theta_{b}} c_{\psi_{b}}-c_{\phi_{b}} s_{\psi_{b}}}{c_{\theta_{b}} c_{\psi_{b}}}\right)
\end{aligned}
$$

From the same modified sequence of rotations, $\dot{\tilde{\psi}}_{p}$ and $\dot{\tilde{\psi}}_{b}$ are given in Eqs. (28) and (29):

$$
\begin{aligned}
& \dot{\tilde{\psi}}_{p}=-c_{\tilde{\psi}_{p}} t_{\tilde{\theta}_{p}} p_{p}+s_{\tilde{\psi}_{p}} t_{\tilde{\theta}_{p}} q_{p}+r_{p} \\
& \dot{\tilde{\psi}}_{b}=-c_{\tilde{\psi}_{b}} t_{\tilde{\theta}_{b}} p_{b}+s_{\tilde{\psi}_{b}} t_{\tilde{\theta}_{b}} q_{b}+r_{b}
\end{aligned}
$$

where

$$
\begin{aligned}
t_{\tilde{\theta}_{p}} & =\frac{c_{\phi_{p}} s_{\theta_{p}} c_{\psi_{p}}+s_{\phi_{p}} s_{\psi_{p}}}{c_{\theta_{p}} c_{\psi_{p}}} c_{\tilde{\psi}_{p}} \\
t_{\tilde{\theta}_{b}} & =\frac{c_{\phi_{b}} s_{\theta_{b}} c_{\psi_{b}}+s_{\phi_{b}} s_{\psi_{b}}}{c_{\theta_{b}} c_{\psi_{b}}} c_{\tilde{\psi}_{b}}
\end{aligned}
$$

Given the state vector of the system, the 12 linear equations in Eq. (4) are solved to obtain derivatives of the state vector along with the coupling joint constraint force components required for numerical simulation.

\section{Results}

The system of equations given in Eq. (4) is solved using LU decomposition and the equations of motion just described are numerically integrated using a fourth-order Runge-Kutta algorithm to generate the trajectory of the system from its point of release. Simulations under different conditions are performed so that the performance of the controllable parafoil and payload system can be evaluated. The payload is a cube measuring $1.0 \mathrm{ft}(0.3 \mathrm{~m})$ on a side and has a weight of $10 \mathrm{lbf}(44 \mathrm{~N})$ with uniform density. The parafoil consists of five panels as shown in Fig. 2, each having dimensions of $1.25 \times 2.5 \mathrm{ft}(0.38 \times 0.76 \mathrm{~m})$ and having a combined weight of $0.5 \mathrm{lbf}(2.2 \mathrm{~N})$. The mass center of each panel from its base is $1.3 \mathrm{ft}(0.4 \mathrm{~m})$. The parafoil panel area remains small from the release of the parafoil until $0.6 \mathrm{~s}$ when the panel areas increase until $2.9 \mathrm{~s}$ when the final areas are reached. The length of the rigid links from the coupling joint to the payload mass center and the coupling joint to the parafoil mass center are $\boldsymbol{r}_{c \rightarrow b}=3.0 \boldsymbol{K}_{b} \mathrm{ft}$ $\left(0.91 \boldsymbol{K}_{b} \mathrm{~m}\right)$ and $\boldsymbol{r}_{c \rightarrow p}=-0.5 \boldsymbol{I}_{p}-4.0 \boldsymbol{K}_{p} \mathrm{ft}(-0.15-1.22 \mathrm{~m})$, respectively. The rotational stiffness and damping at joint $\mathrm{C}$ were chosen to be $0.35 \mathrm{lb} \cdot \mathrm{ft} / \mathrm{rad}(0.47 \mathrm{~N} \cdot \mathrm{m} / \mathrm{rad})$ and $0.025 \mathrm{lb} \cdot \mathrm{ft} / \mathrm{rad}^{2}$ $\left(0.034 \mathrm{~N} \cdot \mathrm{m} / \mathrm{rad}^{2}\right)$, which were sufficient to maintain the parafoil and payload within $10 \mathrm{deg}$ of yaw angle. The panel aerodynamic coefficients used in the simulations are shown in Fig. 4. The generated coefficients are representative of the general parafoil simulated and have the same trends as data collected for parafoils over a broad range of dimensions. ${ }^{8,10,13}$ The six apparent mass coefficients are based of the following formulas of Lissaman and Brown, ${ }^{14}$ where, $t, c$, and $b$ are the thickness, chord, and span of the parafoil. The appropriate air density must multiply the coefficients in Eqs. (32-37).

$$
A=k_{A} \pi\left(t^{2} b / 4\right)
$$


Table 1 Parafoil dimensions

\begin{tabular}{lc}
\hline \hline Parameters & Values \\
\hline$t$ & $0.33 \mathrm{ft}$ \\
$c$ & $2.5 \mathrm{ft}$ \\
$b$ & $6.0 \mathrm{ft}$ \\
$a^{*}$ & 0.17 \\
$A^{*}$ & 2.4 \\
\hline \hline
\end{tabular}

Table 2 Apparent mass and correction coefficients

\begin{tabular}{lcccc}
\hline \hline \multirow{2}{*}{ Correction coefficients } & & \multicolumn{2}{c}{ Apparent } \\
\cline { 5 - 6 } Parameters & Values & & Parameters & Values \\
\hline$k_{A}$ & 0.913 & & $A$ & 0.0001 \\
$k_{B}$ & 0.339 & & $B$ & 0.0002 \\
$k_{C}$ & 0.771 & & $C$ & 0.0466 \\
$k_{A}^{*}$ & 0.630 & & $I_{A}$ & 0.1141 \\
$k_{B}^{*}$ & 0.872 & & $I_{B}$ & 0.0111 \\
$k_{C}^{*}$ & 1.044 & & $I_{C}$ & 0.0033 \\
\hline \hline
\end{tabular}

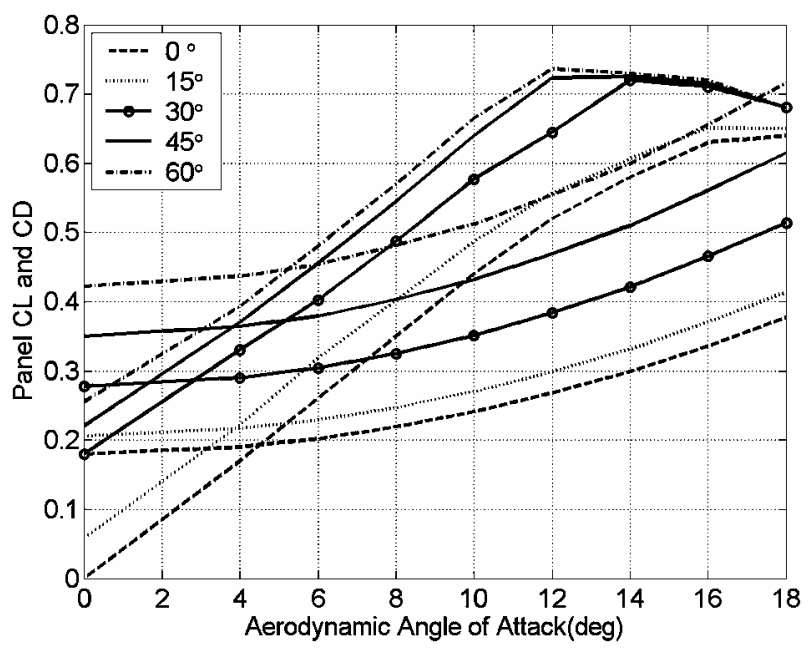

Fig. 4 Lift and drag coefficients for varying brake deflections.

$$
\begin{gathered}
B=k_{B} \pi\left(t^{2} c / 4\right) \\
C=k_{C} \pi\left(c^{2} b / 4\right) \\
I_{A}=k_{A}^{*} \pi\left(c^{2} b^{3} / 48\right) \\
I_{B}=k_{B}^{*}\left(4 c^{4} b / 48 \pi\right) \\
I_{C}=k_{C}^{*} \pi\left(t^{2} b^{3} / 48\right)
\end{gathered}
$$

The apparent mass coefficients in Eqs. (32-37) have threedimensional correction factors that are also given by Lissaman and Brown that depend on the aspect ratio $A^{*}$ and the arc-to-span ratio $a^{*}$. For the properties of the parafoil listed in Table 1, Eqs. (32-37) and the three-dimensionalcorrection factors are evaluated and listed in Table 2.

For the baseline simulation the parafoil and payload system is released from an altitude of $5000 \mathrm{ft}$ with a level speed of $50 \mathrm{ft} / \mathrm{s}$. The panel angles $\alpha_{1}, \alpha_{3}$, as shown in Fig. 2, are 35 and $15 \mathrm{deg}$, respectively; $\alpha_{5}$ is $0 \mathrm{deg}$; and the angle of incidence is $-8.5 \mathrm{deg}$. Baseline simulation results are shown in Figs. 5-10. Figure 5 plots pitch angle vs time of the payload and parafoil, which shows a large negative pitch of the parafoil and payload as a result of the large aerodynamic forces on the payload and the small aerodynamic forces on the parafoil before it fully opens. The opening of the parafoil at $0.6 \mathrm{~s}$ begins an increase in aerodynamic forces on the parafoil, and the pitch angles of both the payload and parafoil begin to increase before settling to $-7.0 \mathrm{deg}$ for the payload and $-29.5 \mathrm{deg}$ for the parafoil. The body pitch rates of the payload and parafoil shown in Fig. 6 oscillate at a frequency of $2 \mathrm{~Hz}$ during the opening of the

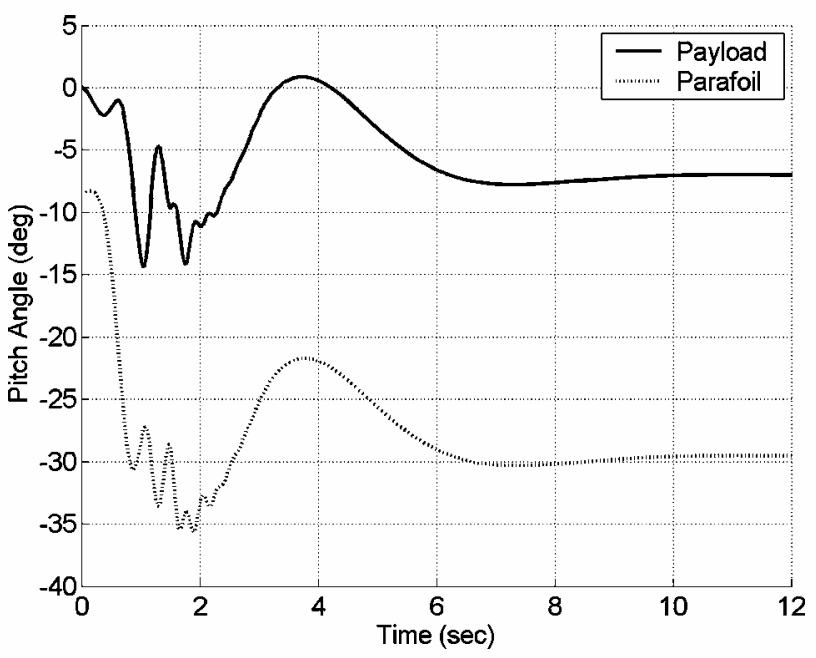

Fig. 5 Pitch angle vs time.

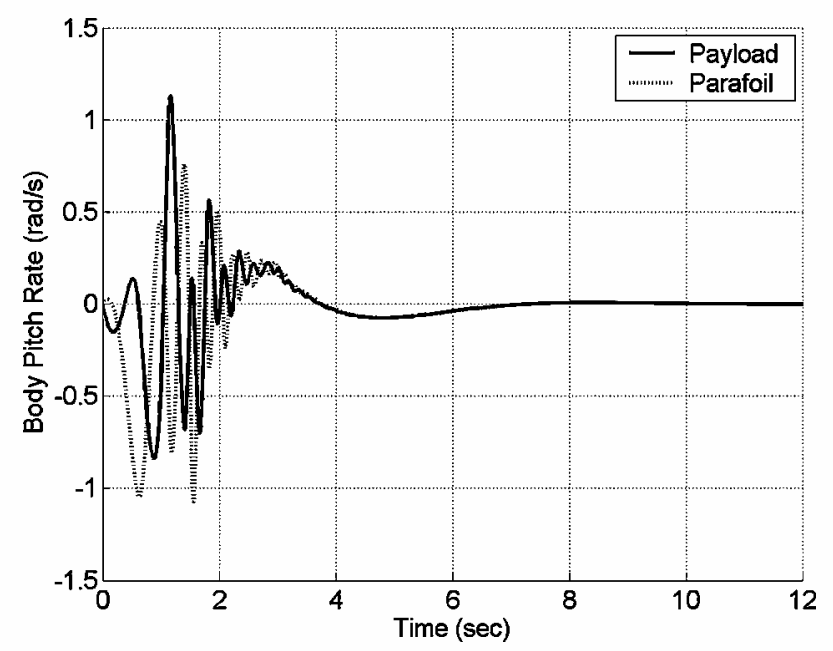

Fig. 6 Body pitch rate vs time.

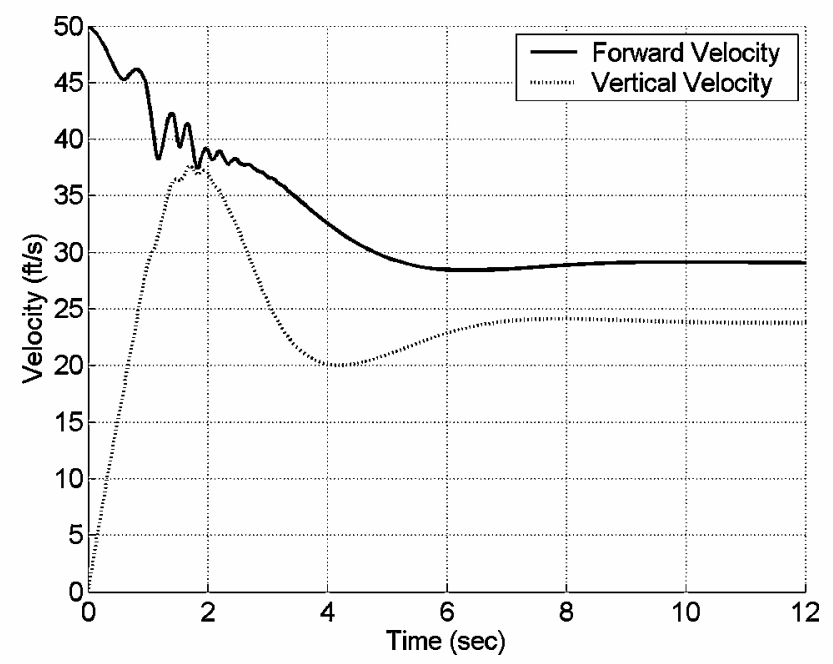

Fig. 7 Velocity vs time.

parafoil at $0.6 \mathrm{~s}$ and decay to near 0 by $12.0 \mathrm{~s}$. The vertical velocity, forward velocity, aerodynamic angle of attack, and constraintforces shown in Figs. 7-9 also show similar oscillatory characteristicsduring the opening of the parafoil and reach steady states by $12.0 \mathrm{~s}$. The altitude of the payload mass center vs time shown in Fig. 10 begins to decrease rapidly during the opening of the parafoil but reaches a steady glide rate after the pitch angle of the payload and parafoil have reached their steady-state values. 


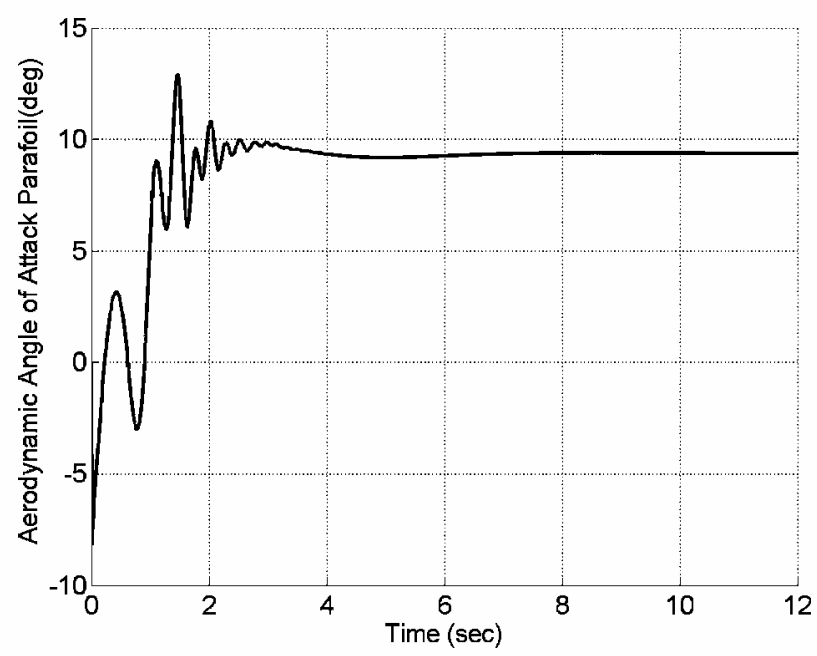

Fig. 8 Aerodynamic angle of attack vs time.

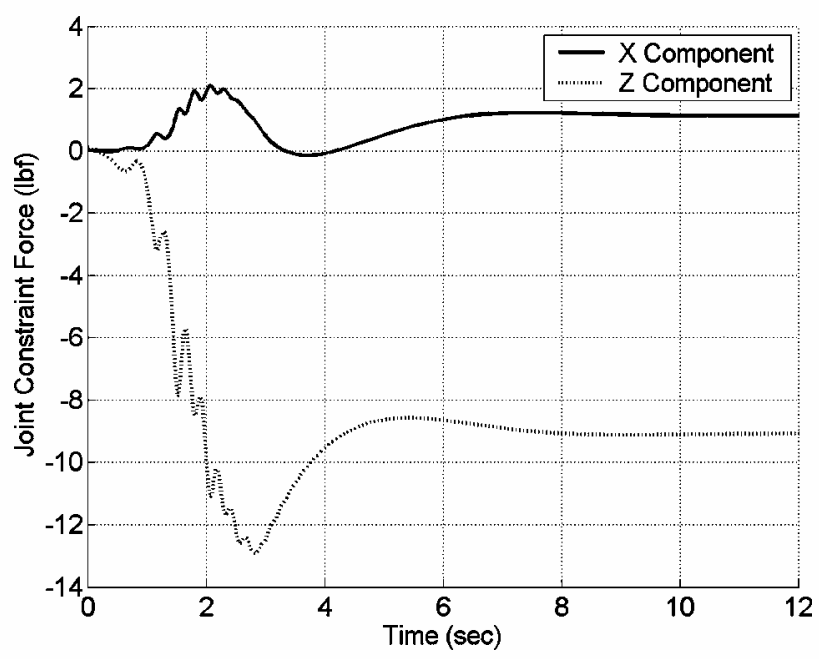

Fig. 9 Constraint forces vs time.

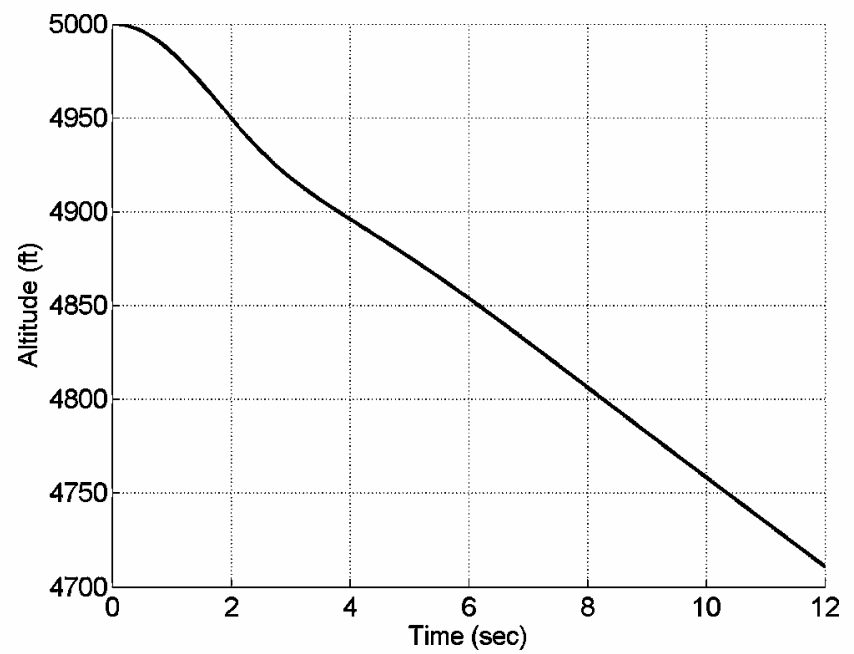

Fig. 10 Altitude vs time.

For a controllable parafoil a subject of interest is the control authority of both large and small brake deflections. The control response to a brake deflection is dependent on the orientation of the panel angles. A set of nine differentcases of panelorientationis used in the following trade studies and is defined in Table 3. Figure 11 shows the response of the baseline parafoil with a -3.0-deg angle of incidence and a constant small right side brake of 10 deg applied after a 10 -s settling period. Cases C, D, and E have negative turn
Table 3 Panel angles

\begin{tabular}{lccccc}
\hline \hline & \multicolumn{5}{c}{ Panel angle, deg } \\
\cline { 2 - 6 } Case & Panel 1 & Panel 2 & Panel 3 & Panel 4 & Panel 5 \\
\hline A & 15 & -15 & 10 & -10 & 0 \\
B & 19 & -19 & 11 & -11 & 0 \\
C & 23 & -23 & 12 & -12 & 0 \\
D & 27 & -27 & 13 & -13 & 0 \\
E & 31 & -31 & 14 & -14 & 0 \\
F & 35 & -35 & 15 & -15 & 0 \\
G & 39 & -39 & 16 & -16 & 0 \\
H & 43 & -43 & 17 & -17 & 0 \\
I & 47 & -47 & 18 & -18 & 0 \\
\hline \hline
\end{tabular}

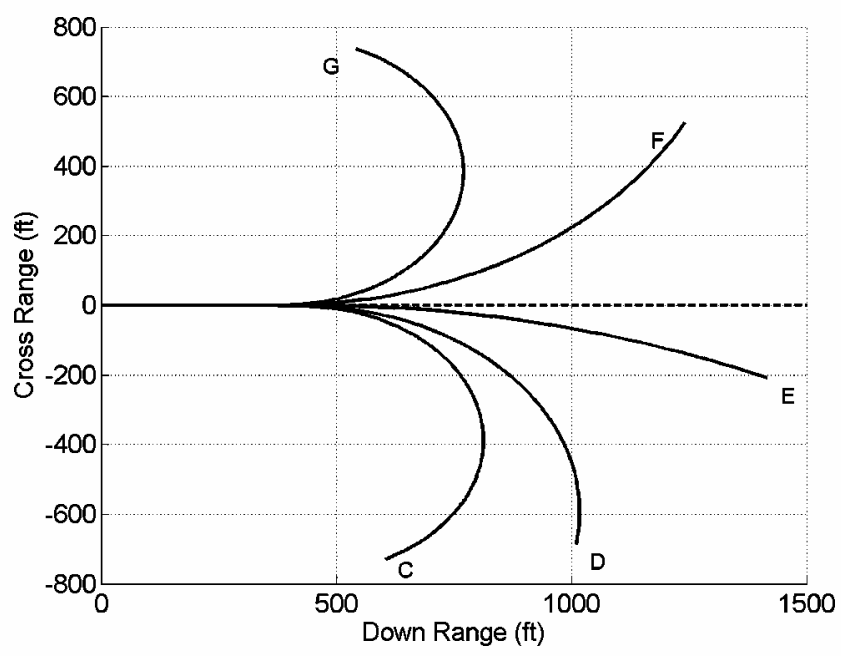

Fig. 11 Cross range vs time (angle of incidence $=-\mathbf{3 . 0} \mathrm{deg}, \mathbf{1 0}-\mathrm{deg}$ right brake).

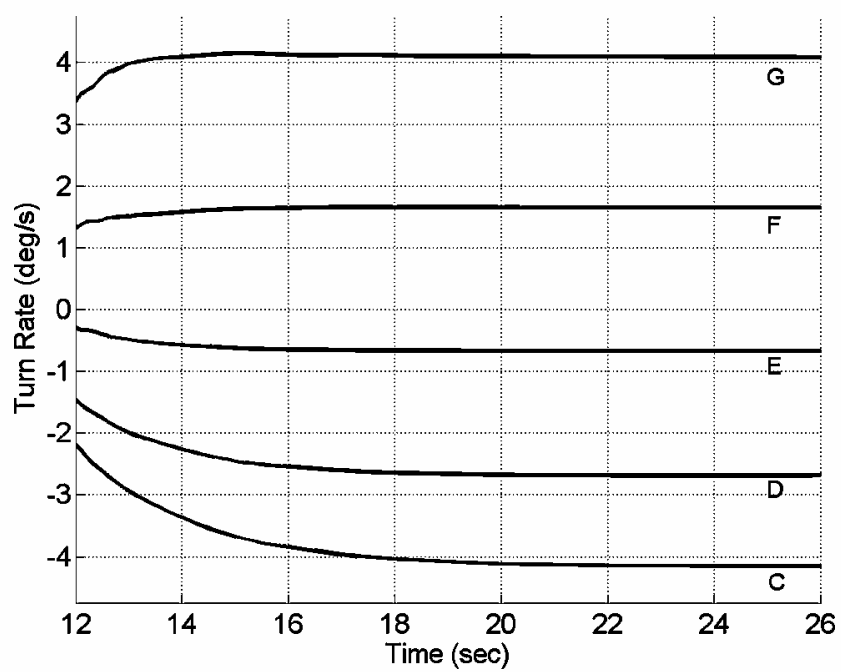

Fig. 12 Turn rate vs time (angle of incidence $=-3.0 \mathrm{deg}, 10-\mathrm{deg}$ right brake).

rates for the small right side brake, whereas cases $F$ and $G$ have positive turn rates. The control authority of small braking reverses as the orientation of the panel angles become more curved. The baseline parafoil with a -3.0-deg angle of incidence demonstrates two modes of control. The mode of control for the less curved cases A-E is roll steering. The flatter parafoil uses increased lift, which dominates drag from the 10-deg brake to roll the parafoil and subsequently yaw. The mode of control for the more curved cases F-I is skid steering. Increased drag dominates lift, and increased drag on the right side of the parafoil generates yawing of the parafoil. Figure 12 shows the turn rates vs time for the five parafoil cases shown in Fig. 11. The negative sign on the turn rate signifies the 
turn is counterclockwise if looking down on the parafoil. It can be seen that the turn rates settle to a near constant value by $22 \mathrm{~s}$ for all five panel cases. A critical panel orientation occurs between cases $\mathrm{E}$ and $\mathrm{F}$ where the parafoil switches from roll steering to skid steering and a small brake would fail to generate yawing. Turn rates are shown in Fig. 13 vs panel case for three angles of incidence: -3.0, -7.0 , and $-13.0 \mathrm{deg}$. The critical panel orientation changes as the angle of incidence is decreased. The critical panel orientation for a -3.0-deg angle of incidence is between cases $\mathrm{E}$ and $\mathrm{F}$, for $-7.0 \mathrm{deg}$ the critical angle is between $\mathrm{F}$ and $\mathrm{G}$, and for -13.0 deg the critical angle is between $\mathrm{G}$ and $\mathrm{H}$. Reducing the angle of incidence or reducing the curvature of the parafoil canopy moves the mode of steering toward roll steer and decreases the control authority of a nominally skid steering parafoil and increases the control authority of a nominally roll steer parafoil. Iacomini and Cerimele observed this trend in NASA's X-38, which is a skid steering parafoil, noting that making the angle of incidence more severe "decreased turn rates for a given turn setting."

To investigate the sensitivity of the control responsecaused by the lift to drag ratio of the parafoil, the drag curves shown in Fig. 4 were held constant while the lift curves were varied $\pm 15 \%$. The control response is dependent on the lift-to-drag ratio of the panels, and the turn rates are shown in Fig. 14 vs steady-state lift-to-drag ratio for three angles of incidence: $-3.0,-7.0$, and $-13.0 \mathrm{deg}$. Similar to varying panel curvature, a critical lift-to-drag ratio occurs where the parafoil switches from roll steering to skid steering and a small break fails to generate yawing. The critical lift-to-drag ratio changes as the angle of incidence is decreased. The critical lift-to-drag ratio for a -3.0-deg angle of incidence is $2.04 \mathrm{deg}$; for $-7.0 \mathrm{deg}$ and $-13.0 \mathrm{deg}$ no critical lift-to-drag ratio is reached, and a skid steering mode does not occur.

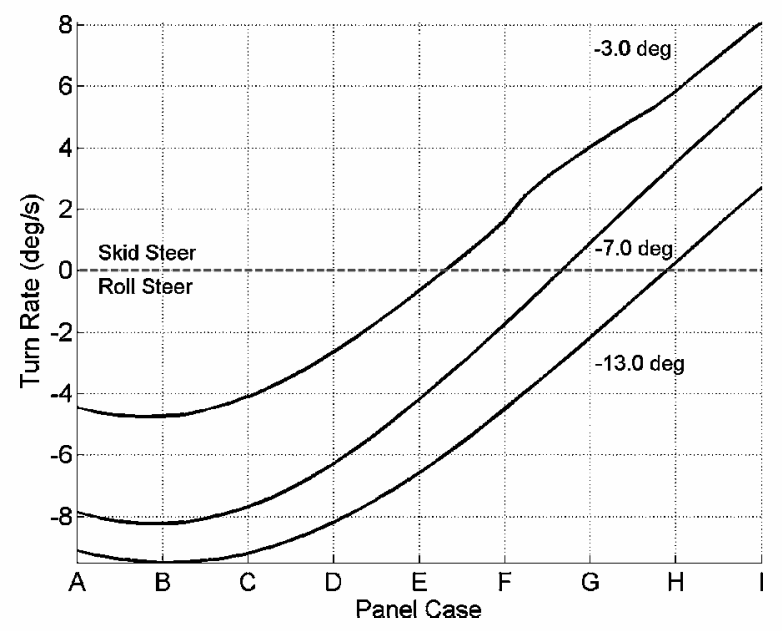

Fig. 13 Turn rate vs panel case (10-deg right brake).

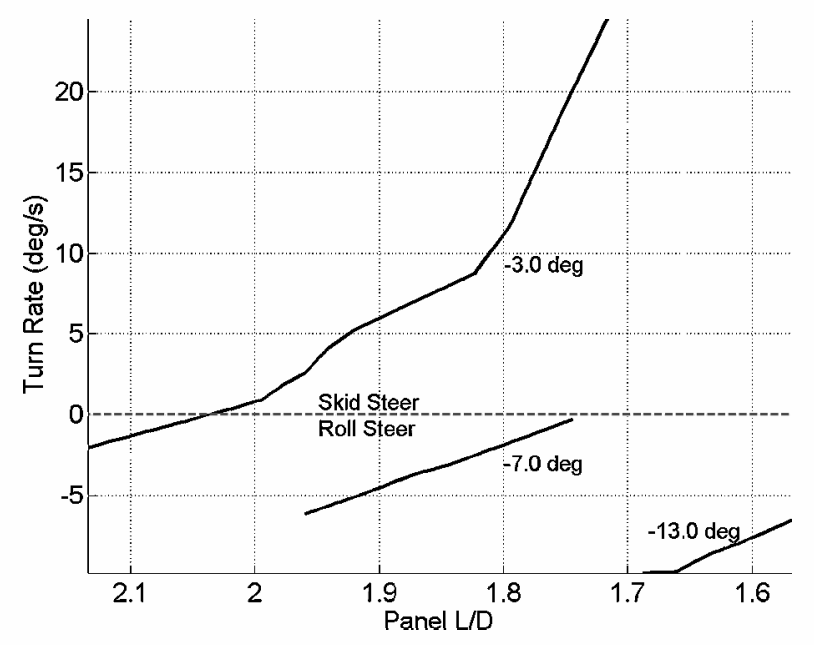

Fig. 14 Turn rate vs lift-to-drag ratio (10-deg right brake).

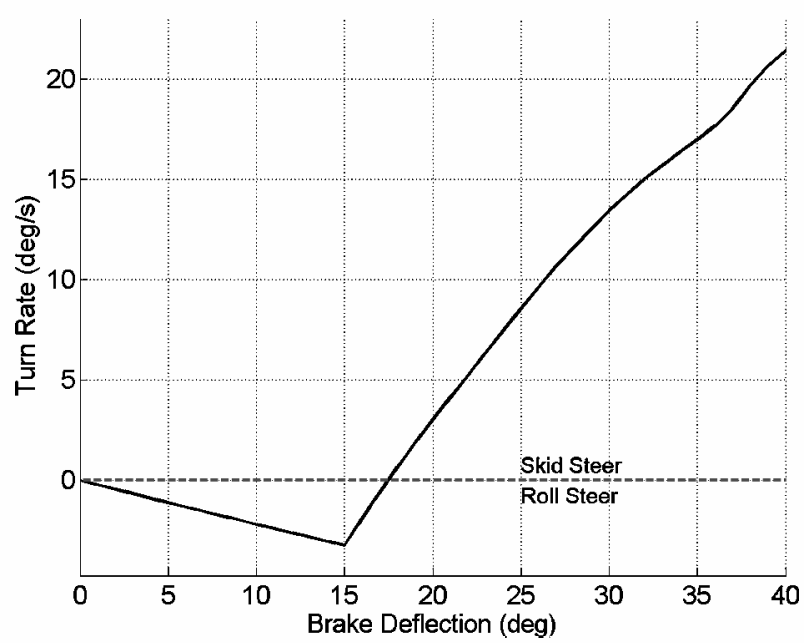

Fig. 15 Turn rate vs brake deflection.

The control authority of the parafoil also depends on the magnitude of the control input. The turn rate is shown vs control input in Fig. 15 for panel case $\mathrm{F}$ and an angle of incidence of $-7.0 \mathrm{deg}$. As shown in Fig. 13, this corresponds to a roll steer mode at small brake deflections. It can be seen in Fig. 15 that the roll steering mode increases its control authority until a brake deflection of $15 \mathrm{deg}$ is reached. After $15 \mathrm{deg}$ the steering transitions toward a skid steering mode as brake deflection is increased until the parafoil reaches skid steering at $17.5 \mathrm{deg}$. The roll steering mode transitions to skid steering as the brake deflection increases and drag begins to dominate. Iacomini and Cerimele have observed, although attributed to brake reflex, the phenomenon of a control reversal for small brake deflections in NASA's X-38 program. ${ }^{10}$ In fact, parafoils mostly operate in a skid steering mode when the brake deflections are large. It should be clear from the trends in Figs. 13 and 15 that a parafoil can be designed to never roll steer even for even the smallest brake deflections. A parafoil that skid steers for all of the brake deflections, curvatures, and angles of incidence however maintains the observed trends, namely, that skid steering control authority is increased as brake deflection is increased, panel curvatureis increased, and the angle of incidence becomes less negative. More importantly, a parafoil that demonstrates roll steering only for small brake deflections such as the parafoil in Fig. 15 can be modified to eliminate all roll steering tendencies by changing the curvature or angle of incidence.

\section{Conclusions}

Using a nine-degree-of-freedom flight dynamic model, it has been shown that parafoil and payload systems exhibit two basic modes of directional control: skid steering and roll steering for small brake deflections. For a particular configuration the mode of directional control depends on the angle of incidence and the panel orientation. The parafoil's mode of directional control is skid steering for canopies of "high" curvature and "smaller" negative angles of incidence. The mode of directional control transitions toward roll steering as the canopy curvature decreases or the angle of incidence becomes more negative. The mode of directional control also transitions away from the roll steering mode as the magnitude of the brake deflection increases, and for "large" brake deflections most parafoils will always skid steer. Control reversal is usually undesirable, and because parafoils have a tendency to skid steer for large brake deflections care needs to be taken to know and avoid the range of small braking that can induce roll steering. With careful design a parafoil and payload system can be properly modified so that roll steering can be eliminated all together.

\section{References}

${ }^{1}$ Wolf, D., "Dynamic Stability of Nonrigid Parachute and Payload System," Journal of Aircraft, Vol. 8, No. 8, 1971, pp. 603-609.

${ }^{2}$ Doherr, K., and Schilling, H., "9 DOF-Simulation of Rotating Parachute Systems," AIAA Paper 91-0877, April 1991. 
${ }^{3}$ Hailiang, M., and Zizeng, Q., "9-DOF Simulation of Controllable Parafoil System for Gliding and Stability," Journal of National University of Defense Technology, Vol. 16, No. 2, 1994, pp. 49-54.

${ }^{4}$ Iosilevskii, G., "Center of Gravity and Minimal Lift Coefficient Limits of a Gliding Parachute," Journal of Aircraft, Vol. 32, No. 6, 1995, pp. 1297-1302.

${ }^{5}$ Brown, G. J., "Parafoil Steady Turn Response to Control Input," AIAA Paper 93-1241, May 1993.

${ }^{6}$ Zhu, Y., Moreau, M., Accorsi, M., Leonard, J., and Smith, J., "Computer Simulation of Parafoil Dynamics," AIAA Paper 2001-2005, May 2001.

${ }^{7}$ Gupta, M., Xu, Z., Zhang, W., Accorsi, M., Leonard, J., Benney, R., and Stein, K., "Recent Advances in Structural Modeling of Parachute Dynamics," AIAA Paper 2001-2030, May 2001.

${ }^{8}$ Ware, G. M., and Hassell, J. L., Jr., "Wind-Tunnel Investigation of
Ram-Air_Inflated All-Flexible Wings of Aspect Ratios 1.0 to 3.0," NASA TM SX-1923,1969.

${ }^{9}$ Iacomini, C. S., and Cerimele, C. J., "Lateral-Directional Aerodynamics from a Large Scale Parafoil Test Program," AIAA Paper 99-1731, Nov. 1999.

${ }^{10}$ Iacomini, C. S., and Cerimele, C. J., "Longitudinal Aerodynamics from a Large Scale Parafoil Test Program,” AIAA Paper 99-1732, Nov. 1999.

${ }^{11}$ McCormick, B., Aerodynamics and Aeronautics and Fight Mechanics, Wiley, New York, 1979, pp. 61-151.

${ }^{12}$ Wolfe, W. P., and Peterson, C. W., "Modeling of Parachute Line Wrap," AIAA Paper 2001-2030, May 2001.

${ }^{13}$ Ellison, R. K., "Determination of a Parafoil Submunition Payload and Aerodynamic Control," Aeromechanics Div., Air Force Armament Lab., AFATL-TR-85-74, Eglin AFB, FL, July 1986.

${ }^{14}$ Lissaman, P. B. S., and Brown, G. J., "Apparent Mass Effects on Parafoil Dynamics," AIAA Paper 93-1236, May 1993. 